the most frequent cause was complex febrile seizures (57\%); epilepsies accounted for $22 \%$, and CNS infections (5\%). The most commonly used treatments for CSE in Japan are diazepam, phenytoin, and barbiturates as $1^{\text {st }}, 2^{\text {nd }}$, and $3^{\text {rd }}$-line drugs. Midazolam i.v has recently become a more favorable $2^{\text {nd }}$ line drug. (Sugai K. Acta Neurol Scand 2007;115 (s186):62-70).

Natsume and colleagues findings of hippocampal pathology following prolonged febrile seizures provide further evidence of risk of mesial temporal sclerosis (MTS) and temporal lobe epilepsy (TLE) as late complications of childhood febrile seizures. Scott RC et al postulated that MTS nay develop after a lag period (Scott RC et al. Brain 2003;126:25512557; ibid Epilepsia 2006;47:1493-1498; Ped Neur Briefs Oct 2006;20:77). Neurosurgical experience has shown that adult patients with TLE associated with MTS are more likely to have a childhood history of febrile seizures than those without MTS (Falconer MA. Epilepsia 1971;12:13-31). Perhaps the "benign" nature of febrile seizures has been overemphasized. Efforts to prevent recurrence and rapidly abort febrile seizures should be given more attention, especially in patients with a first complex febrile seizure.

\title{
COGNITIVE DISORDERS IN BENIGN CHILDHOOD EPILEPSY WITH CENTROTEMPORAL SPIKES (BECTS)
}

Intelligence and language functions were examined in 24 children (mean age 9 yrs; range 7-12 yrs) with BECTS and compared with a group of 16 controls matched for age and schooling, in a study at the Instituto Nazionale Neurologico, Milan, Italy. Tests measuring phonemic fluency, verbal expression of semantic knowledge, and reading comprehension revealed mild language defects. Neuropsychological functions were impaired by excess interictal EEG discharges while awake, multifocal spike location, and temporal location. A side-specific impairment was noted, with deficiencies in phonemic fluency correlating with left-sided spikes, and impaired lexical comprehension with right-sided spikes. (Riva D, Vago $\mathrm{C}$, Franceschetti $\mathrm{S}$ et al. Intellectual and language findings and their relationship to EEG characteristics in benign childhood epilepsy with centrotemporal spikes. Epilepsy Behav March 2007;10:278-285). (Respond: Dr Daria Riva, Developmental Neurology Division, Instituto Nazionale Neurologico C Besta, Via Celoria 11, Milan, Italy).

COMMENT. Children with BECTS have a slightly lower IQ and subtle language impairments compared to controls. A high spike frequency in the waking state has negative effects on cognitive and language function, whereas the sleeping spike rate shows no correlation. In this study, the BECTS-associated subtle neuropsychological dysfunction is not reflected in any school difficulties. Findings at reexamination in later childhood, when BECTS has resolved, will be of interest.

\section{ENDOCRINE EFFECTS OF VALPROATE IN ADOLESCENT GIRLS WITH EPILEPSY}

The effects of epilepsy and/or valproate (VPA) monotherapy on physical growth, weight gain, pubertal development, and hormonal status of 68 consecutive female patients aged 6-20 years (20 premenarche, 60 postmenarche) were studied at the Institute for 
Endocrinology and Diabetes, Schneider Children's Medical Center, and Tel Aviv University, Israel. Two groups of patients, 45 during long-term treatment with VPA and 43 before treatment was initiated, were compared. Treated postmenarcheal patients had a higher mean testosterone level than untreated controls $(\mathrm{p}=0.006)$. Body mass index-standard deviation scores were not increased. Rates of obesity were not significantly different in treated and untreated groups, $16.3 \%$ and $15.5 \%$, respectively. Menses irregularities, hirsutism, and acne occurred with equal frequency in the 2 groups. The treated group had higher levels of thyroid-stimulating hormone and lower levels of free thyroxine than the untreated group, but both were within the normal range. (de Vries L, Karasik A, Landau Z et al. Endocrine effects of valproate in adolescent girls with epilepsy. Epilepsia March 2007;48:470-477). (Reprints: Dr H Goldberg-Stern, Epilepsy Center, Schneider Children's Medical Center of Israel, 14 Kaplan St, Petah Tiqwa, 49202 Israel).

COMMENT. Long-term treatment with valproate in girls with epilepsy results in increased testosterone levels after menarche, without signs of hyperandrogenism, polycystic ovary syndrome, or increase in body mass index. Endocrine function should still be monitored if valproate is prescribed for epilepsy in adolescent girls. Other studies have linked obesity, hyperinsulinemia, hyperandrogenism, and polycystic ovaries with long-term valproate therapy for epilepsy in women (Isojarvi JIT et al. Ann Neurol 1996;39:579-584). Epileptic and neurologic factors, and not valproate, have been cited as the primary cause of polycystic ovary syndrome (Hertzog AG. Ann Neurol 1996;39:559-560). Valproate should be avoided if possible in women of childbearing age and during pregnancy, given the high rate of associated fetal malformations, neonatal neurobehavioral and late neurological side effects (Koch S et al. Acta Paediatr 1996;84:739-746).

\section{INFECTIOUS DISORDERS}

\section{NEUROLOGIC SEQUELAE OF ENTEROVIRUS 17 INFECTION}

The long-term neurologic sequelae, neurodevelopment, and cognitive function of 142 children with a history of enterovirus (EV) 17 infection with CNS involvement were determined in a study at the National Taiwan University Hospital, Taipei; and Chang Gung Children's Hospital, Taoyuan, Taiwan. The median age of disease onset was 1.8 years (range 0.1 to 13.5), and age at time of assessment was 5.0 years (range 1.3 to 20.8). Sixty-one had aseptic meningitis, 53 had severe CNS involvement, and 28 had cardiopulmonary failure after the CNS involvement. Limb weakness and atrophy developed in $9(56 \%)$ of 16 patients with a poliomyelitis-like syndrome, and in $1(20 \%)$ of 5 with encephalomyelitis. Of the 28 with cardiopulmonary failure following CNS involvement, $18(64 \%)$ had limb weakness and atrophy, $17(61 \%)$ required tube feeding, and $16(57 \%)$ required ventilator support. Children with the complication of cardiopulmonary failure had a significantly higher incidence of delayed neurodevelopment and lower IQ scores than children with CNS involvement alone. Scores on the Denver Development Screening Test for children 6 years of age or younger were delayed in 21 of $28(75 \%)$ with cardiopulmonary failure cf to 1 of $20(5 \%)$ with severe CNS involvement alone $((\mathrm{P}<0.001)$; the mean full-scale IQ on the WISC Test for children 4 years of age or older was lower in patients with cardiopulmonary failure $(\mathrm{P}=0.003)$. (Chang L-Y, Huang L-M, Gau SS-F et al. Neurodevelopment and cognition in children after 